\title{
GINS2 affects cell proliferation, apoptosis, migration and invasion in thyroid cancer via regulating MAPK signaling pathway
}

\author{
SAIFEI HE $^{1 *}$, MIAO ZHANG $^{1 *}$, YING YE $^{1}$, YANAN SONG $^{1}$, XING MA $^{2}$, \\ GUOYU WANG ${ }^{2}$, JUHUA ZHUANG ${ }^{2}$, WEI XIA ${ }^{2 *}$ and BIN ZHAO ${ }^{3 *}$ \\ ${ }^{1}$ Central Laboratory; Departments of ${ }^{2}$ Nuclear Medicine and ${ }^{3}$ General Surgery, \\ Seventh People's Hospital of Shanghai University of Traditional Chinese Medicine, Shanghai 200137, P.R. China
}

Received November 12, 2019; Accepted November 30, 2020

DOI: $10.3892 / \mathrm{mmr} .2021 .11885$

\begin{abstract}
Globally, thyroid cancer (TC) is considered to be the commonest endocrine malignancy. GINS complex subunit 2 (GINS2) belongs to the GINS complex family and is associated with cellular migration, invasion and growth. The present study aimed to investigate the underlying mechanisms of GINS2 on cell viability, migration and invasion in TC cells. By using MTT, wound healing and Transwell assays, the cell viability, migration and invasion were determined. Apoptosis was examined by immunofluorescence. Western blotting was used to detect protein expression levels. In the present study, biological function analysis demonstrated that GINS2 interference attenuated cell viability, migration and invasion in TC cell lines (K1 and SW579). It was discovered that, compared with the control group, GINS2 silencing induced apoptosis in TC cells. Additionally, GINS2 interference inhibited key proteins in the MAPK signaling pathway, including JNK, ERK and p38. According to these comparative experiments, GINS2 was considered to act a pivotal part in cell viability, migration and invasion of TC by regulating the MAPK signaling pathway and might be a potential therapeutic target for treating TC.
\end{abstract}

\section{Introduction}

Thyroid cancer (TC) is the most frequent endocrine malignancy in the world $(1,2)$. Over the last decade, the incidence rate of

Correspondence to: Professor Wei Xia, Department of Nuclear Medicine, Seventh People's Hospital of Shanghai University of Traditional Chinese Medicine, 358 Datong Road, Pudong, Shanghai 200137, P.R. China

E-mail: awingxia@163.com

Professor Bin Zhao, Department of General Surgery, Seventh People's Hospital of Shanghai University of Traditional Chinese Medicine, 358 Datong Road, Pudong, Shanghai 200137, P.R. China E-mail: qiyuanzb@163.com

*Contributed equally

Key words: GINS complex subunit 2, thyroid cancer, cell viability, migration and invasion, MAPK signaling pathway
TC incidence has been increasing by $\sim 2 \%$ per year, with a slow but steady increase in the mortality rate $(\sim 0.7 \%$ per year) (3). There are several risk factors that are closely associated with TC occurrence and development, including genetic factors, age, sex, environmental exposure and epigenetic alterations. Even though there have been improvements in the diagnosis and surgical treatment of TC, the disadvantages associated with treatment strategies should not be ignored $(4,5)$. Furthermore, TC demonstrates an increasing morbidity year on year, along with high rate of recurrence and a younger age at diagnosis, indicating a requirement for additional research on TC (3). Therefore, it is necessary to elucidate the underlying mechanisms associated with the progression and pathogenesis of TC and in turn help its treatment.

Sld5, Psf1 (GINS1), Psf2 (GINS2) and Psf3 (GINS3) constitute the DNA replication complex (6-8). Among them, GINS2 is an important subunit of GINS DNA replication complex and is located on human chromosome at 16q24 (9). The relative molecular mass of GINS2 is $21 \mathrm{kDa}$ and the mRNA length is $1,196 \mathrm{bp}(8,10,11)$. GINS2 mediates the interaction between MCM complexes and Cdc45 during the initiation of DNA replication in eukaryotic cells (9). Previous reports have identified that GINS2 is associated with a number of malignant tumors $(12,13)$. It mainly affects the initiation and progression of malignancy. For example, the expression of GINS2 is remarkably upregulated in acute promyelocytic leukemia $(14,15)$. In addition, upregulation of GINS2 is found in lung cancer tissues and is in turn associated with cancer metastasis (16). GINS2 can enhance the ability of growth and proliferation in cervical cancer (6). As a consequence, GINS2 could act as a potential prognosis indicators and drug target in some types of malignant tumors. The connection between GINS2 and the development of TC requires further elucidation.

MAPK belongs to serine/threonine protein kinases $(17,18)$. The MAPK signaling cascade acts as a critical pathway for tumor cell proliferation, differentiation, apoptosis and drug-resistance $(19,20)$. MAPK is activated by a variety of stimulants including cytokines, growth factors, neurotransmitters and hormones $(21,22)$. In addition, MAPK activation can phosphorylate nuclear transcription factors and protein kinases, regulate the transcription of related genes and participate in various physiological processes (22). Previous 
studies have shown that the MAPK signaling pathway is activated in a various types of cancer, including gastric (23), lung (24), ovarian (25) and liver cancers (26). The MAPK signaling pathway consists of four sub-pathways: ERK, JNK, BMK and p38 (27). Studies have shown that activation of the ERK signaling pathway facilitates cell growth, differentiation, migration and survival $(28,29)$. Activation of JNK and p38 involves changes of cell differentiation, apoptosis and cell survival (30). However, the function and regulation of BMK1/ERK5 have been explored by fewer studies although this pathway has been reported in the regulation of cell growth, differentiation and survival (31).

The present study aimed to investigate the relationship between GINS2 and TC and explore the effects of MAPK signaling pathway in the process of GINS2 that affects TC cells, offering a new potential diagnosis target for TC.

\section{Materials and methods}

Cell lines and authentication. TC cell lines (K1 and SW579) were obtained from The Cell Bank of Type Culture Collection of The Chinese Academy of Sciences, Shanghai, China. K1 cells were maintained in $5 \% \mathrm{CO}_{2}$ at $37^{\circ} \mathrm{C}$ with RPMI-1640 medium (HyClone; Cytiva) supplemented with $10 \%$ fetal bovine serum (10\% FBS; Gibco; Thermo Fisher Scientific, Inc.) and penicillin/streptomycin (HyClone; Cytiva). SW579 cells were maintained in $0 \% \mathrm{CO}_{2}$ at $37^{\circ} \mathrm{C}$ with L-15 medium (HyClone; Cytiva) supplemented with 10\% FBS and penicillin/streptomycin. In order to ensure the accuracy of results, STR profiling was performed for K1 cells. DNA was extracted with genomic extraction kit (Axygen; Corning, Inc.) and amplified with 21-STR. Data were analyzed using an ABI 3730XL genetic analyzer (Applied Biosystems; Thermo Fisher Scientific, Inc.). The EXPASY database (www.expasy. org/) confirmed that the cell name was K1 and the cell number corresponded to CVCL_2537. No multiple allelic groups were found in the cell line.

Gene interference. Small interfering (si)RNA was used to knock down specific sequences. The oligonucleotides against GINS2 sequences were designed and synthesized by Shanghai GenePharma Co., Ltd. The following sequences were included: GINS2 siRNA (sense: GCUCAACCACAUGUACAAATT and antisense: UUUGUACAUGUGGUUGAGCTT); and negative control (NC) sequence (sense: UUCUCCGAACGU GUCACGUTT and antisense: ACGUGACACGUUCGG AGAATT). These are not homologous to any human DNA sequences. K1 and SW579 cells were seeded into culture plate and incubated at $37^{\circ} \mathrm{C}$ overnight, followed by transfection with siRNA-GINS2, blank and respective controls (80 nM) using Lipofectamine ${ }^{\circledR} 2000$ (Invitrogen; Thermo Fisher Scientific, Inc.) according to the manufacturer's protocols. After 24-48 h transfection, the subsequent experiments were performed.

Western blot analysis. Total proteins were isolated from cell lysates using RIPA buffer containing protease inhibitors and phosphatase inhibitors (Beyotime Institute of Biotechnology). The protein concentrations were determined using a BCA protein assay kit (Beyotime Institute of Biotechnology). Protein $(\sim 40 \mu \mathrm{g})$ from each sample was separated to $10 \%$ SDS-PAGE and then transferred onto a PVDF membrane (EMD Millipore). The membranes were washed with $\mathrm{pH} 7.5,50 \mathrm{mM}$ Tris-HCL buffer saline containing $0.05 \%$ Tween-20 (TBST) and blocked with $5 \%$ skimmed milk powder for $1 \mathrm{~h}$ at room temperature. The PVDF membrane was incubated with primary antibodies at $4^{\circ} \mathrm{C}$ overnight with gentle agitation. The following primary antibodies were used in the present study: GINS2 $(1: 1,000$; cat. no. ab197123; Abcam), ERK (1:1,000; cat. no. 4695; Cell Signaling Technology, Inc.), phosphorylated (p-)ERK (1:2,000; cat. no. 4370; Cell Signaling Technology, Inc.), JNK (1:1,000; cat. no. 9252; Cell Signaling Technology, Inc.), p-JNK (1:2,000, cat. no. 9255, Cell Signaling Technology, Inc.), p38 (1:1,000; cat. no. 8690; Cell Signaling Technology, Inc.), p-p38 (1:1,000; cat. no. 4511; Cell Signaling Technology, Inc.), and GAPDH (1:1,000; cat. no. 5174; Cell Signaling Technology, Inc.). The samples were washed with TBST (containing $0.1 \%$ Tween-20), then incubated with secondary antibodies conjugated with horseradish peroxidase (1:3,000; cat. nos. 7074 and 7076; Cell Signaling Technology, Inc.) at room temperature for $1.5 \mathrm{~h}$. The blots were observed using an enhanced chemiluminescence detection kit (Beyotime Institute of Biotechnology). The levels of the proteins of interest were normalized to GAPDH and analyzed using FluorChem FC3 software (version 3.4.0, ProteinSimple).

MTT assay. Cell proliferation was measured using MTT assay according to the manufacturer's protocols (Beyotime Institute of Biotechnology). The TC cell lines were cultivated in 96-well plates at a density of $\sim 5 \times 10^{3} /$ well, followed by treatment with siGINS2 at 12, 24, 48 and 72 h. CCK- 8 reagent $(10 \mu l)$ was added to each well and incubation for $4 \mathrm{~h}$ at $37^{\circ} \mathrm{C}$. The optical density (OD) value of each well was measured at $450 \mathrm{~nm}$ by using a microplate reader (Promega Corporation). The cell viability results from three independent experiments were normalized to the medium control group and expressed as the mean \pm standard deviation.

Apoptosis assay. Cell apoptosis rate was detected by a fluorescein isothiocyanate (FITC)-Annexin V/propidium iodide (PI) apoptosis kit (Beyotime Institute of Biotechnology). TC cells were seeded into a 24 -well plate at a density of $\sim 2 \times 10^{5}$ cells/well for apoptosis assay. Following treatment with NC sequence or GINS2 siRNA, the cells were washed twice with PBS, followed by addition of Annexin V-FITC (5 $\mu \mathrm{l}$ ) into the well according to the manufacturer's protocols and maintained for $15 \mathrm{~min}$ at room temperature for the reaction to take place. Propidium iodide $(10 \mu \mathrm{l})$ was added into the tube and left for $15 \mathrm{~min}$ at room temperature. Cell apoptosis was determined by using an inverted fluorescence microscope (Olympus Corporation) within $1 \mathrm{~h}$. Data were analyzed using ImageJ 1.46 (National Institutes of Health).

Transwell assay. A Transwell 24-well Boyden chamber (8 $\mu \mathrm{m}$ pore size, Corning, Inc.) with or without Matrigel was used to determine the capacity of migration and invasion. For migration assay, the cells after treatment were digested with $0.25 \%$ trypsin (Thermo Fisher Scientific, Inc.) and then suspended in serum-free medium. The cells were counted and seeded at a density of $1 \times 10^{5}$ cells/well in the upper chamber. A total of $700 \mu \mathrm{l}$ of complete medium was 
A

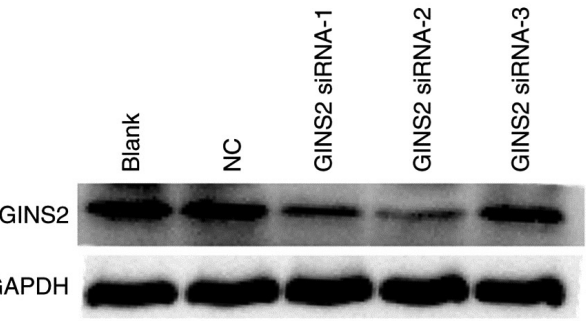

K1

C
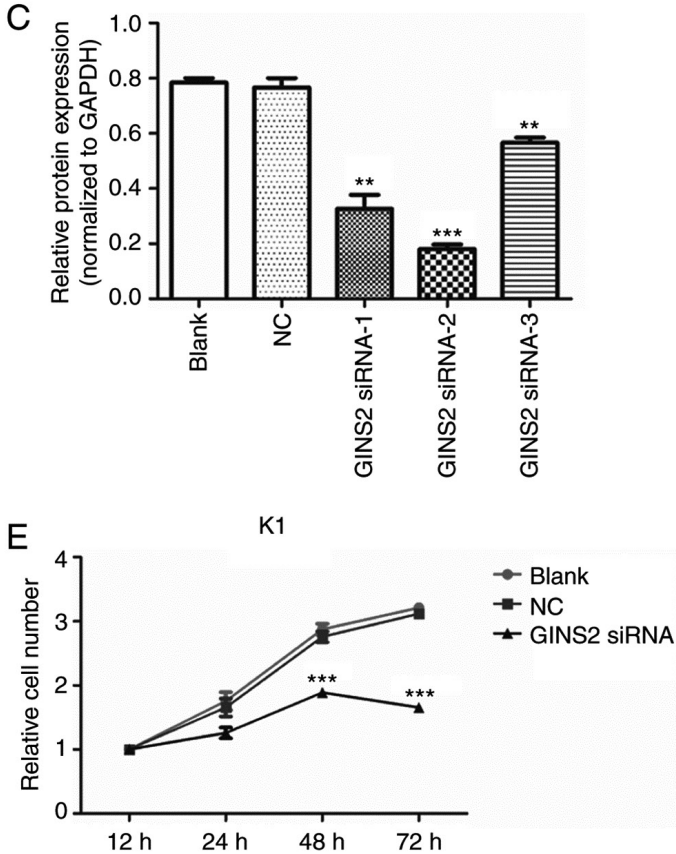

B

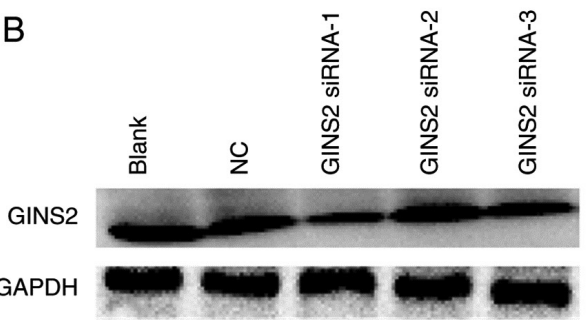

SW579

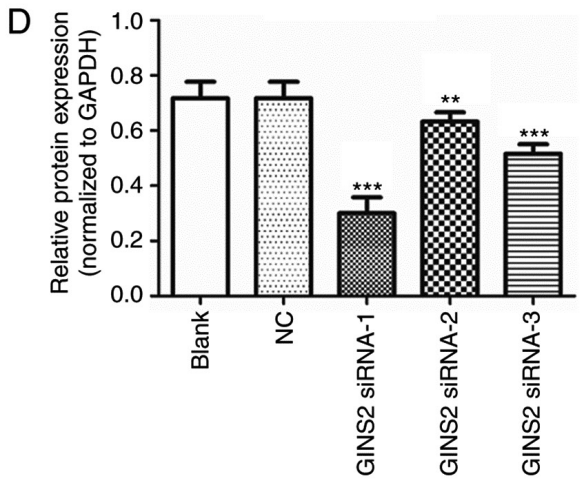

F

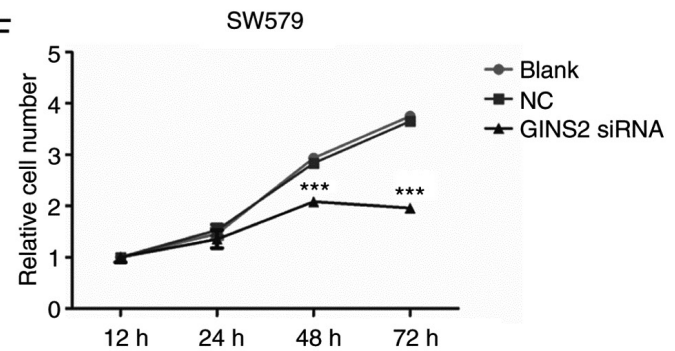

Figure 1. Suppression of GINS2 decreases the cell viability in TC cells. (A and C) K1 and (B and D) SW579 were transfected with Blank, NC or siGINS2 for $48 \mathrm{~h}$ and then the protein expression of GINS2 was determined by western blotting. GAPDH was used as an internal standard. Cell viability was estimated by using MTT assay in (E) K1 and (F) SW579 at 12, 24, 48 and 72 h. The absorbance was measured at OD $450 \mathrm{~nm}$ using a microplate reader. All experiments were performed at least three times. Data are presented as the means \pm standard deviation. ${ }^{* *} \mathrm{P}<0.01$ and ${ }^{* * *} \mathrm{P}<0.001$ vs. NC using one-way ANOVA followed by Tukey's test. GINS2, GINS complex subunit 2; TC, thyroid cancer; NC, negative control; si, small interfering; OD, optical density.

placed in the lower chamber as a source of chemo-attractant. After $24 \mathrm{~h}$, the non-migratory cells in the upper layer of the chamber were removed, while the cells which had migrated through the membrane were fixed with $4 \%$ paraformaldehyde for $10 \mathrm{~min}$ and stained with $0.5 \%$ crystal violet for $30 \mathrm{~min}$ at room temperature. A total of five random high power fields were observed and images captured under a light microscope (magnification, x100, Olympus Corporation), then the migration through the membrane calculated as the average number of cells/field. For invasion assay, the steps were similar to that of the cell migration, with the only difference being that the Transwell membranes were precoated with Matrigel for 30 min at $37^{\circ} \mathrm{C}$ (diluted at 1:2; BD Biosciences).

Wound healing assay. A wound healing assay was used to assess the cell migration ability in TC cell lines. Following the transfection of K1 and SW579 cells, the cells were trypsinized and seeded into a 6-well plate and cultured until they reached $80 \%$ confluence in a complete medium. Each well was then scratched by a $200 \mu 1$ sterile pipette tip and washed with PBS several times to remove cell debris. In the next $48 \mathrm{~h}$, the cells were incubated with serum-free medium and the cells that migrated to the wound surface were considered as producing an in vitro healing process. Images of the wound healing were captured under a light microscope (magnification, x100; Olympus Corporation) and the rate of closure was assessed. The relative migratory ability was evaluated using ImageJ 1.46 (National Institutes of Health) based on the width at $0 \mathrm{~h}$ time point (the wound width of (0-48 h)/0 h wound width $\mathrm{x} 100 \%$.

Statistical analysis. All the data are shown as means \pm standard deviation and analyzed by one-way analysis of variance followed by Tukey's test. Statistical analyses were performed using SPSS v16.0 software (SPSS, Inc.). The experiments were repeated $\geq 3$ times. $\mathrm{P}<0.05$ was considered to indicate a statistically significant difference.

\section{Results}

Suppression of GINS2 markedly inhibits the cell proliferation of TC cells. K1 and SW579 cells were treated with GINS2 siRNA to inhibit the expression of GINS2. Fig. 1A and C (K1 cells) and Fig. 1B and D (SW579 cells) show the western blotting images of GINS2 interference by different siRNAs. The most effective sequence (GINS2 siRNA-1) was selected to conduct further experiments. To examine whether GINS2 has an effect 
A
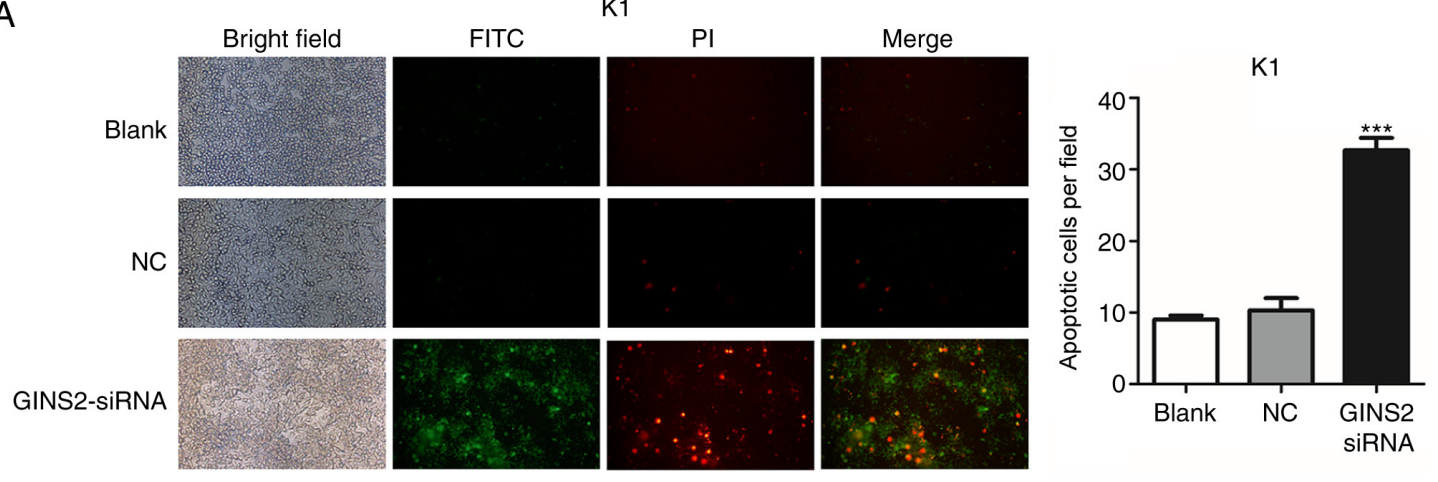

B
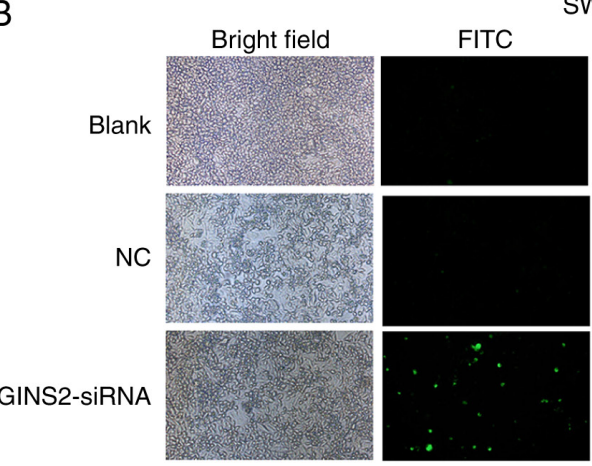

SW579
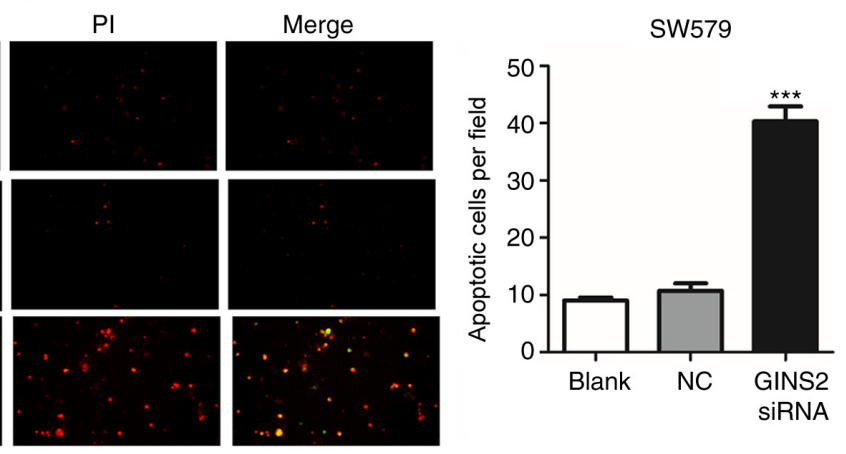

Figure 2. Suppression of GINS2 causes apoptosis in TC cells. Annexin V-FITC and PI staining were performed to evaluate apoptosis in (A) K1 and (B) SW579 cells. Annexin V-FITC stained cells demonstrated green fluorescence and PI stained of cells demonstrated red fluorescence. The cells in green fluorescence were apoptotic cells, those in green and red fluorescence were necrotic cells and those not stained with green fluorescence were normal cells. Magnification, $\mathrm{x} 100$. Data are presented as the means \pm standard deviation. ${ }^{* * *} \mathrm{P}<0.001$ vs. NC using one-way ANOVA followed by Tukey's test. GINS2, GINS complex subunit 2; TC, thyroid cancer; FITC, fluorescein isothiocyanate; PI, propidium iodide; NC, negative control; si, small interfering.

on TC cell proliferation, MTT assay was performed. TC cells were transfected with GINS2 siRNA and incubated for 12, 24, 48 and $72 \mathrm{~h}$. The results demonstrated that the absorbance in K1 (Fig. 1E) and SW579 (Fig. 1F) cells was increased from 12 to $72 \mathrm{~h}$ in the control group, while the OD $450 \mathrm{~nm}$ value was increased from 12 to $48 \mathrm{~h}$ and decreased from 48 to $72 \mathrm{~h}$ in the GINS2 siRNA group. From 24 to $72 \mathrm{~h}$, the number of TC cells in the GINS2 siRNA group was clearly lower compared with the control $(\mathrm{P}<0.001)$. These results demonstrated that cell viability was significantly inhibited following silencing of the GINS2 gene by siRNA in K1 and SW579 cells.

Suppression of GINS2 induces cell apoptosis in TC cells. Whether GINS2 interference could affect cell apoptosis in K1 and SW579 cells was studied. The cells were subjected to Annexin V-FITC/PI staining. The results revealed that the GINS2 suppression induced apoptotic cell death of TC cells (Fig. 2A and B), suggesting that inhibition of GINS2 initiated cell death.

Suppression of GINS2 decreases migration and invasion of TC cells. The ability of migration and invasion is a key indicator of tumor metastasis. The present study used wound healing and Transwell assays to determine the capacity of tumor metastasis. The results of wound healing assay demonstrated that silencing of GINS2 significantly inhibited the migratory capacity in K1 and SW579 cells (P<0.01; Fig. 3A-D). In addition, Transwell assay with or without Matrigel was used to investigate the effect of GINS2 on the migratory and invasive abilities of K1 and SW579 cells. As demonstrated in Fig. 3E-H, interference of GINS2 significantly suppressed the migratory and invasive abilities of K1 and SW579 cells $(\mathrm{P}<0.001)$. These results suggested that GINS2 acted as a promoter gene of migration and invasion in TC cells.

Suppression of GINS2 affects TC proliferation through the MAPK signaling pathway. The forgoing results demonstrated that downregulation of GINS2 caused changes in the physiological function of the cells, including cell proliferation, apoptosis, migration and invasion. To determine the mechanism between GINS2 and TC, signaling molecules of the MAPK pathway associated with proliferation and migration (ERK, JNK and p38) were detected by western blotting. This demonstrated that the phosphorylation levels of ERK, JNK and p38 were significantly decreased with GINS2 interference in K1 and SW579 cell lines when compared to control cells $\left({ }^{* * *} \mathrm{P}<0.001\right.$; Fig. 4A-F). These results indicated that downregulation of GINS2 inhibited over-activated MAPK signaling pathways in TC cells.

\section{Discussion}

The present study presented a comprehensive investigation on the role of GINS2 in regulating TC tumorigenesis. Functional analyses demonstrated that silencing of GINS2 inhibited cell proliferation, migration and invasion and induced cell apoptosis in TC cell lines. Furthermore, GINS2 interference affected the biological function of TC cells by regulating the 

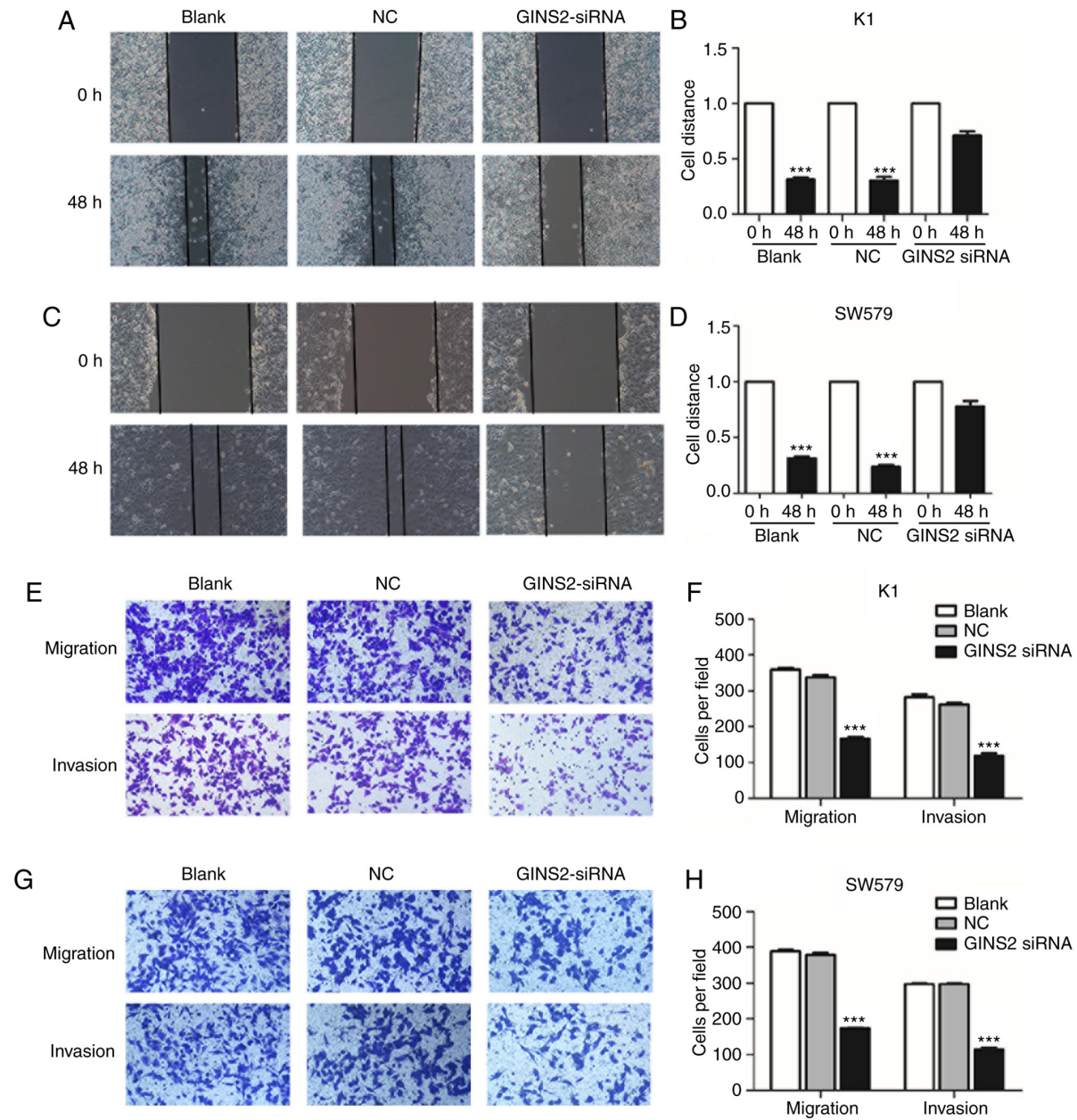

Figure 3. Suppression of GINS2 decreases migration and invasion of TC cells. (A-D) Cell migration activity was measured by conducting the wound healing assay $48 \mathrm{~h}$ following transfection. (E-H) For cellular migration and invasion abilities, Transwell assays were performed on TC cells. Magnification, x100. Data are presented as the means \pm standard deviation for experiments conducted thrice. ${ }^{* * *} \mathrm{P}<0.001 \mathrm{vs}$. NC using one-way analysis of variance followed by Tukey's test. GINS2, GINS complex subunit 2; TC, thyroid cancer; NC, negative control; si, small interfering.

A
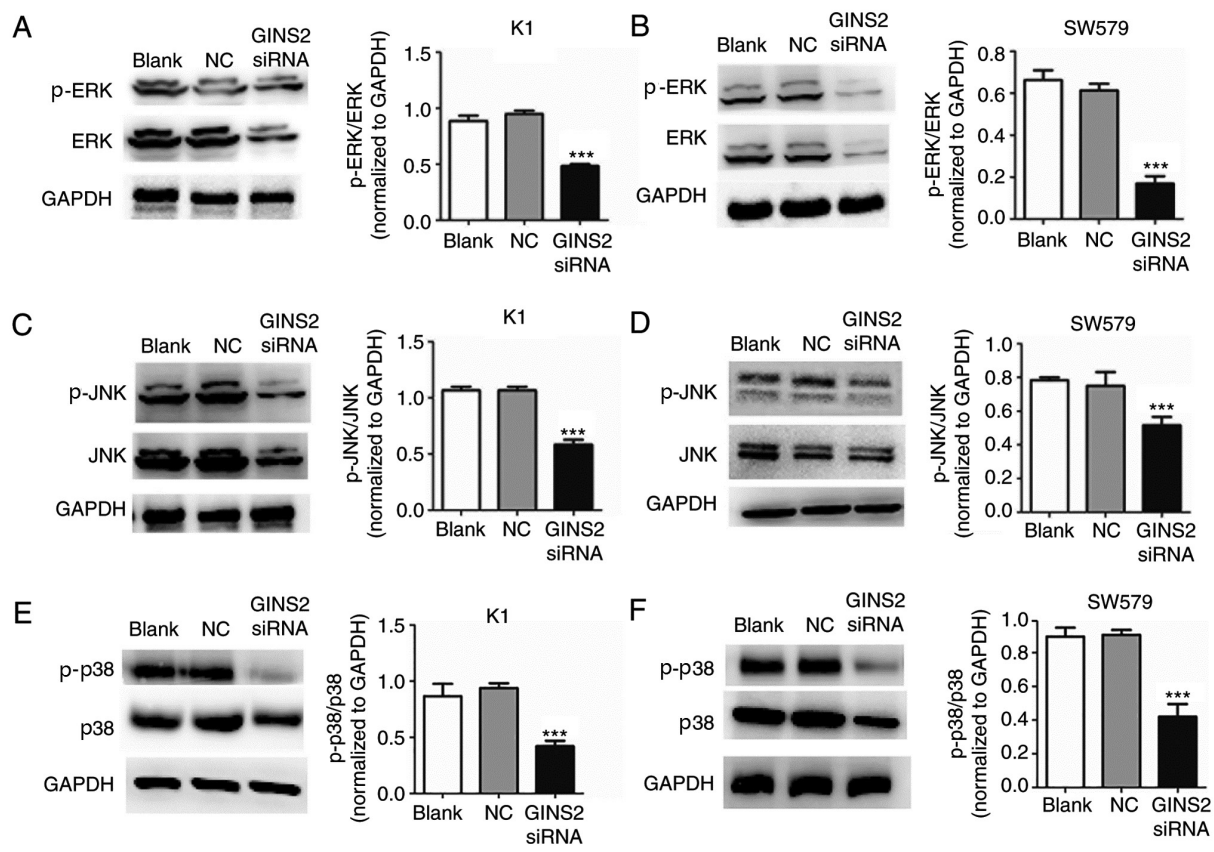

Figure 4. Suppression of GINS2 inhibits the MAPK signaling pathway. Following treatment of cells with NC-vector or GINS2-siRNA, the expression levels of phosphorylated ERK, JNK and p38 in (A, C and E) K1 and (B, D and F) SW579 cells were respectively confirmed by western blotting. GAPDH was used as endogenous control. Data are expressed as means \pm standard deviation for experiments conducted thrice. ${ }^{* * * *} \mathrm{P}<0.001$ vs. NC using one-way ANOVA followed by Tukey's test. GINS2, GINS complex subunit 2; NC, negative control; si, small interfering; p- phosphorylated. 
MAPK signaling pathway. These results implied that GINS2 might be a potential therapeutic target for treating TC.

Overproliferation, migration and invasion of tumor cells are important problems that urgently require clarification and resolution in the clinical and experimental research of malignant tumors and this is also the case in thyroid cancer $(32,33)$. Changes in the physiological functions of tumor cells is a complex process that involves multiple genes and pathways. GINS is a ring-like protein complex that involves PSF1, PSF2 (GINS2), PSF3 and SLD5 and is initially extracted from budding yeast (34). GINS2 acts as an important subunit of GINS complexes and mediates the initiation of DNA replication in eukaryotic cells $(35,36)$. Previous studies have revealed that GINS2 is associated with the malignancy of some cancer types and is highly expressed in several malignant tumors $(6,12)$, including TC (9). Furthermore, overexpression of GINS2 demonstrates an association with prognostic survival period and distant metastasis (12).

Previous experimental and clinical studies have shown that GINS2 is closely associated with tumorigenesis $(6,12)$ and the present study hypothesized that GINS2 might be involved in tumor malignancy. Cell proliferation, migration and invasion are regarded as the most important biological characteristics of malignant cell behavior (13). Tumor cells have the characteristics of limitless replicative potential, tissue invasion and metastasis, resisting cell death (37). In particular, limitless proliferation of tumor cells promotes the progression of metastasis, which is one of the major causes of poor prognosis (24). In the present study, GINS2 silencing resulted in reduced cell proliferation, decreased migration rate and impaired invasive ability in TC cells. Furthermore, cell apoptosis was increased in K1 and SW579 cells following GINS2 silencing by in situ fluorescence detection. Previous studies have demonstrated that in situ fluorescence assay can verify the change of apoptosis rate $(38,39)$. Alternatively, caspase-3 activity and other techniques can also be applied for cell apoptosis assay, which will be performed in further studies.

The results of the present study confirmed that GINS2 might serve an important role in TC progression and the related molecular mechanisms in this process require further investigation. Cells encounter a variety of signals in their environment and respond to each stimulus appropriately by modulating gene and protein expression expression levels $(17,26)$. Numerous external signals are transduced by a highly conserved eukaryotic signaling mechanism, including the well-known MAPK cascade (20). MAPK, which is a type of serine/threonine protein kinase that can be activated by different extracellular stimuli, including cytokines, neurotransmitters, hormones, cell stress and cell adhesion, is an important transporter of signals from cell surface to nucleus (17). MAPK regulates cell proliferation, differentiation, development and apoptosis through signaling transmission $(23,40)$. In our previous study, it was found that GINS2 interference inhibited cell viability, induced cell cycle arrest and promoted cell apoptosis in pancreatic cancer cell lines via the MAPK/ERK pathway (41). In the present study, the relationship between GINS2 and MAPK pathway was investigated in TC cells. The results demonstrated that GINS2 silencing resulted in significantly decreased expression levels of phosphorylated ERK, JNK and p38 in K1 and SW579 cells.
These results indicated that silencing of GINS2 could inhibit the activation of MAPK pathway and then affect proliferation, apoptosis, migration and invasion of cells.

However, there are still some limitations in the present study. Rescue assays were not performed, wherein MAPK was overexpressed at the same time as GINS2 downregulation, in order to investigate if MAPK overexpression can overcome the effect of GINS2 knockdown. Also, GINS2 may also inhibit or activate other signaling pathways, which will be further investigated in the future.

The present study preliminarily clarified that GINS2 silencing suppressed cell proliferation, migration and invasion and induced cell apoptosis in TC by regulating MAPK signaling pathways. More studies should be conducted to illuminate the function of GINS2 and its related mechanisms in the future.

\section{Acknowledgements}

Not applicable.

\section{Funding}

The present study was supported by grants from National Natural Science Foundation of China (grant nos. 81873178 and 81904044), Shanghai Municipal Commission of Health and Family Planning (grant no. 201740084), Science and Technology Development Fund of Shanghai Pudong New Area (grant no. PKJ2019-Y15), Key Specialty Construction Project of Pudong Health and Family Planning Commission of Shanghai (grant no. PWZzk2017-21), Shanghai Pudong Commission of Health and Family Planning (grant no. PW2017B-11) and Talents Training Program of Seventh People's Hospital of Shanghai University of TCM (grant nos. XX2017-06 and XX2019-01).

\section{Availability of data and materials}

The datasets used and/or analyzed during the current study are available from the corresponding author on reasonable request.

\section{Authors' contributions}

WX and BZ conceived the experiments. SH, MZ, XM and GW conducted the experiments. YY, YS and JZ analyzed the data. $\mathrm{MZ}$ and YS wrote and revised the paper. WX and BZ confirm the authenticity of the data used in the present manuscript. All authors read and approved the final manuscript.

\section{Ethics approval and consent to participate}

Not applicable.

\section{Patient consent for publication}

Not applicable.

\section{Competing interests}

The authors declare that they have no competing interests. 


\section{References}

1. Xiao Y, Zhou Q, Xu Y, Yuan SL and Liu QA: Positive thyroid antibodies and risk of thyroid cancer: A systematic review and meta-analysis. Mol Clin Oncol 11: 234-242, 2019.

2. Xing M: Molecular pathogenesis and mechanisms of thyroid cancer. Nat Rev Cancer 13: 184-199, 2013.

3. Bonjoc KJ, Young H, Warner S, Gernon T, Maghami E and Chaudhry A: Thyroid cancer diagnosis in the era of precision imaging. J Thorac Dis 12: 5128-5139, 2020.

4. Reale C, Russo F, Credendino SC, Cuomo D, De Vita G, Mallardo M,Pennino F, Porreca I, Triassi M, De Felice M, et al: A toxicogenomic approach reveals a novel gene regulatory network active in in vitro and in vivo models of thyroid carcinogenesis. Int J Environ Res Public Health 16: 16, 2019.

5. Samimi H,Haghpanah V, Irani S, Arefian E, Sohi AN, Fallah P and Soleimani M: Transcript-level regulation of MALAT1-mediated cell cycle and apoptosis genes using dual MEK/Aurora kinase inhibitor 'BI-847325' on anaplastic thyroid carcinoma. Daru 27: $1-7,2019$.

6. Ouyang F, Liu J, Xia M, Lin C, Wu X, Ye L, Song L, Li J, Wang J, Guo P, et al: GINS2 is a novel prognostic biomarker and promotes tumor progression in early-stage cervical cancer. Oncol Rep 37: 2652-2662, 2017

7. Yan T, Liang W, Jiang E, Ye A, Wu Q and Xi M: GINS2 regulates cell proliferation and apoptosis in human epithelial ovarian cancer. Oncol Lett 16: 2591-2598, 2018.

8. Peng L, Song Z, Chen D, Linghu R, Wang Y, Zhang X, Kou X, Yang $\mathbf{J}$ and Jiao S: GINS2 regulates matrix metallopeptidase 9 expression and cancer stem cell property in human triple negative breast cancer. Biomed Pharmacother 84: 1568-1574, 2016.

9. Ye Y, Song YN, He SF, Zhuang JH, Wang GY and Xia W: GINS2 promotes cell proliferation and inhibits cell apoptosis in thyroid cancer by regulating CITED2 and LOXL2. Cancer Gene Ther 26: 103-113, 2019.

10. Liu C, Wang R and Zhang Y: GINS complex subunit 2 (GINS2) plays a protective role in alcohol-induced brain injury. Artif Cells Nanomed Biotechnol 47: 1-9, 2019.

11. Zheng M, Zhou Y, Yang X, Tang J, Wei D, Zhang Y, Jiang JL, Chen ZN and Zhu P: High GINS2 transcript level predicts poor prognosis and correlates with high histological grade and endocrine therapy resistance through mammary cancer stem cells in breast cancer patients. Breast Cancer Res Treat 148: 423-436, 2014

12. Rantala JK, Edgren H, Lehtinen L, Wolf M, Kleivi K, Vollan HK, Aaltola AR, Laasola P, Kilpinen S, Saviranta P, et al: Integrative functional genomics analysis of sustained polyploidy phenotypes in breast cancer cells identifies an oncogenic profile for GINS2 Neoplasia 12: 877-888, 2010

13. Shen YL, Li HZ, Hu YW, Zheng L and Wang Q: Loss of GINS2 inhibits cell proliferation and tumorigenesis in human gliomas. CNS Neurosci Ther 25: 273-287, 2019.

14. Gao Y, Wang S, Liu B and Zhong L: Roles of GINS2 in K562 human chronic myelogenous leukemia and NB4 acute promyelocytic leukemia cells. Int J Mol Med 31: 1402-1410, 2013.

15. Zhang X, Zhong L, Liu BZ, Gao YJ, Gao YM and Hu XX: Effect of GINS2 on proliferation and apoptosis in leukemic cell line. Int J Med Sci 10: 1795-1804, 2013.

16. MacNeill SA: Structure and function of the GINS complex, a key component of the eukaryotic replisome. Biochem J 425: 489-500, 2010.

17. Kim EK and Choi EJ: Compromised MAPK signaling in human diseases: An update. Arch Toxicol 89: 867-882, 2015.

18. Sun J and Nan G: The mitogen-activated protein kinase (MAPK) signaling pathway as a discovery target in stroke. J Mol Neurosci 59: 90-98, 2016.

19. Burotto M, Chiou VL, Lee JM and Kohn EC: The MAPK pathway across different malignancies: A new perspective. Cancer 120: 3446-3456, 2014

20. Gyurkó MD, Steták A, Sőti C and Csermely P: Multitarget network strategies to influence memory and forgetting: The Ras/MAPK pathway as a novel option. Mini Rev Med Chem 15: 696-704, 2015

21. Cuadrado A and Nebreda AR: Mechanisms and functions of $\mathrm{p} 38$ MAPK signalling. Biochem J 429: 403-417, 2010.
22. Ma Y,Zhao Q, Shao Y, Cao MZ, Zhao M and Wang D: Melatonin inhibits the inflammation and apoptosis in rats with diabetic retinopathy via MAPK pathway. Eur Rev Med Pharmacol Sci 23 (Suppl): 1-8, 2019

23. Shang H, Cao Z, Zhao J, Guan J, Liu J, Peng J, Chen Y, Joseph Sferra T, Sankararaman S and Lin J: Babao Dan induces gastric cancer cell apoptosis via regulating MAPK and NF- $\kappa \mathrm{B}$ signaling pathways. J Int Med Res 47: 5106-5119, 2019.

24. Tang G, Zeng Z, Sun W, Li S, You C, Tang F, Peng S, Ma S, Luo Y, $\mathrm{Xu}$ J, et al: Small nucleolar RNA 71A promotes lung cancer cell proliferation, migration and invasion via MAPK/ERK pathway. J Cancer 10: 2261-2275, 2019.

25. Yasui H, Kajiyama H, Tamauchi S, Suzuki S, Peng Y, Yoshikawa N, Sugiyama M, Nakamura K and Kikkawa F: CCL2 secreted from cancer-associated mesothelial cells promotes peritoneal metastasis of ovarian cancer cells through the P38-MAPK pathway. Clin Exp Metastasis 37: 145-158, 2020.

26. Jin C, Chen Z, Shi W and Lian Q: Tropomodulin 3 promotes liver cancer progression by activating the MAPK/ERK signaling pathway. Oncol Rep 41: 3060-3068, 2019.

27. Wagner EF and Nebreda AR: Signal integration by JNK and p38 MAPK pathways in cancer development. Nat Rev Cancer 9: 537-549, 2009

28. Kim EK and Choi EJ: Pathological roles of MAPK signaling pathways in human diseases. Biochim Biophys Acta 1802: 396-405, 2010.

29. Lavoie H, Gagnon J and Therrien M: ERK signalling: A master regulator of cell behaviour, life and fate. Nat Rev Mol Cell Biol 21: 607-632, 2020.

30. Semba T, Sammons R, Wang X, Xie X, Dalby KN and Ueno NT: JNK signaling in stem cell self-renewal and differentiation. Int J Mol Sci 21: 21, 2020.

31. Yu Z, Ye S, Hu G, Lv M, Tu Z, Zhou K and Li Q: The RAF-MEK-ERK pathway: Targeting ERK to overcome obstacles to effective cancer therapy. Future Med Chem 7: 269-289, 2015.

32. Zhou C, Yang C and Chong D: E-cadherin expression is associated with susceptibility and clinicopathological characteristics of thyroid cancer: A PRISMA-compliant meta-analysis. Medicine (Baltimore) 98: e16187, 2019.

33. Kawai T, Iwata K, Shinotsuka Y, Kubo S, Masuoka H, Yabuta T, Hirokawa M,Nakamura H, Miyauchi A and Komai K: CD44v8-10 and CD44s are age-dependently expressed in primary cultured papillary thyroid carcinoma cells and are associated with cell proliferation. Kobe J Med Sci 65: E1-E9, 2019.

34. Hashimoto Y, Puddu F and Costanzo V: RAD51- and MRE11-dependent reassembly of uncoupled CMG helicase complex at collapsed replication forks. Nat Struct Mol Biol 19: $17-24,2011$

35. Moiseeva T, Hood B, Schamus S, O'Connor MJ, Conrads TP and Bakkenist CJ: ATR kinase inhibition induces unscheduled origin firing through a Cdc7-dependent association between GINS and And-1. Nat Commun 8: 1392, 2017.

36. Chmielewski JP, Henderson L, Smith CM and Christensen TW: Drosophila Psf2 has a role in chromosome condensation. Chromosoma 121: 585-596, 2012.

37. Pennati M, Cimino-Reale G, Gatti L and Cassinelli G: Strategies to strike survival networks in cancer. Crit Rev Oncog 21: 269-308, 2016

38. Duan Z, Chen Q, Du L, Tong J, Xu S, Zeng R, Ma Y, Chen X and $\mathrm{Li}$ M: Phagocytosis of Candida albicans inhibits autophagic flux in macrophages. Oxid Med Cell Longev 2018: 4938649, 2018.

39. Chen H, Lin W, Lin P, Zheng M, Lai Y, Chen M, Zhang Y, Chen J, Lin X, Lin L, et al: IL-10 produces a dual effect on OGD-induced neuronal apoptosis of cultured cortical neurons via the NF- $x \mathrm{~B}$ pathway. Aging (Albany NY) 11: 10796-10813, 2019.

40. Roberts PJ and Der CJ: Targeting the Raf-MEK-ERK mitogen-activated protein kinase cascade for the treatment of cancer. Oncogene 26: 3291-3310, 2007.

41. Zhang M, He S, Ma X, Ye Y, Wang G, Zhuang J, Song Y and Xia W: GINS2 affects cell viability, cell apoptosis, and cell cycle progression of pancreatic cancer cells via MAPK/ERK pathway. J Cancer 11: 4662-4670, 2020.

This work is licensed under a Creative Commons Attribution-NonCommercial-NoDerivatives 4.0 International (CC BY-NC-ND 4.0) License. 